\title{
CHARACTERIZING THE DEPARTURE PROCESS FROM A TWO SERVER MARKOVIAN QUEUE: A NON-RENEWAL APPROACH
}

\author{
Guy L. Curry \\ Natarajan Gautam \\ Industrial and Systems Engineering \\ Texas A\&M University \\ College Station, TX 77843-3131, USA
}

\begin{abstract}
For large queueing network analysis the general computational approach is to utilize decomposition to facilitate computational tractability. To accomplish this individual analysis the input and output streams must be characterized. This usually is done via two-parameter characterizations: the process mean and a variance measure (most commonly the squared coefficient of variation SCV). In most approaches independent and identically distributed (i.i.d.) approximations are used. For multiple input streams and/or multiple (identical) servers, the assumptions of i.i.d. times between arrivals and, similarly, i.i.d. times between departures are particularly theoretically and computationally inaccurate. In this paper we develop a generator for the background multidimensional continuous time Markov chain associated with the inter-departure times for the associated multi-stream and multi-server Markovian queues (where inter-arrival times and service times are Coxian). This generator allows for the computation of the moments of the departure process and the lag- $k$ correlations between successive $k$-separated departures.
\end{abstract}

\section{INTRODUCTION}

The semiconductor industry consists of extremely long and complex manufacturing processes. Typically several hundred processing steps are involved in developing the products. These products are manufactured via multiple layered production processes where they make many passes through a sequence of similar processing steps. With the high cost high value of the resulting products it is important to continue analyzing these production systems. Simulation is a very useful and accurate analysis tool. However, it is often fruitful to develop analytical models of these systems and sub-processes. The most widely utilized analytical tool in semiconductor fab systems analysis is queueing network modeling. While analytical models are usually less accurate then simulation models they have a significant advantage in speed. The improvement of the accuracy of the queueing network analytical techniques is an important aspect to their utility. To effectively develop an analytical model of a complex network of production processes such as those encountered in the semiconductor industry, it is necessary to utilize a modeling approach of decomposing the complex interconnected system of processing workstations. The decomposition approach treats each workstation individually by characterization of the inflow and outflow streams. Therefore, it is paramount that we have accurate characterizations of these product flow streams. In this paper we are addressing the characterization of the output flow stream for multiple server workstations. We develop a non-renewal (non i.i.d.) characterization of the output process which will contribute to more accurate analytical systems models.

The main goal of this paper is to develop a characterization of the departure process for Markovian queueing systems with two distinct arrival processes and two identical servers. The system being modeled is assumed to have two independent arrival streams each modeled as a two-phase exponential process with a given probability of using the second phase (sometimes called a Coxian 2 distribution). Even though the arrival processes are independent, the time between arrivals are correlated. In a similar fashion the service processes are independent (but identical) Coxian 2 processes. The inter-departure times of jobs leaving the system are also correlated.

In the standard network node approximation approach, the departure process from a workstation system is normally approximated by assuming that these inter-departure times are independent and identically distributed (i.i.d.). The mean time between arrivals and departures are the same. However, this i.i.d. assumption allows for a simple approximation (Whitt 1983) of the SCV of the departure process $\left(C_{d}^{2}\right)$ as a function of the systems utilization $(\mathrm{u})$ 
and the arrival and service processes SCV's $\left(C_{a}^{2}, C_{s}^{2}\right)$ for a $G / G / m$ queue as

$$
C_{d}^{2}=\left(1-u^{2}\right)\left(C_{a}^{2}-1\right)+u^{2}\left(C_{s}^{2}-1\right) / \sqrt{m}
$$

Bitran and Dasu (1994) developed a phase distribution representation of the departure process from a single server system and provided moments of the inter-departure time of a $\sum \mathrm{Ph}_{\mathrm{i}} / \mathrm{Ph} / 1$ queue We extend those results in two ways. First we consider the multi-server case and second we obtain not only the moments of the inter-departure time but also correlations between successive departures. One of the motivations to use lag coefficients is to combine with matching moments (which arguably is not an effective technique if used in isolation) to characterize the arrivals to a downstream node in the network setting. We do not explicitly address that issue in this paper but it will be included in a forthcoming journal article.

\section{MODEL}

We consider a single station of a queueing network and model the system as a $\sum \mathrm{Ph}_{\mathrm{i}} / \mathrm{Ph} / \mathrm{C}$ queue. For ease of explanation we present the case of two independent arrival streams and two identical servers. The arrival processes are assumed to be independent Coxian 2's with parameters: $\left(\lambda_{1}, \alpha, \lambda_{2}\right),\left(\gamma_{1}, \beta, \gamma_{2}\right)$ (note that these are nonidentical processes). The phases corresponding to the first arrival stream is depicted in Figure 1. The time spent in the first phase is $\exp \left(\lambda_{1}\right)$. Then either an arrival occurs with probability $(1-\alpha)$ or with probability $\alpha$ the process jumps to the second phase where it stays for $\exp \left(\lambda_{2}\right)$ time and then an arrival occurs.

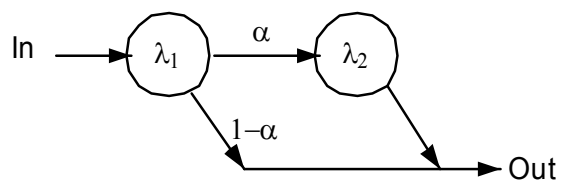

Figure 1: Coxian arrival process (number 1)

Both arrival processes are Coxian distributed inter-arrival times denoted by $\left(\underline{e}_{1}^{T}, T_{i}, T_{i o}\right)$ and characterized by

$$
\begin{array}{ll}
T_{1}=\left[\begin{array}{cc}
-\lambda_{1} & \alpha \lambda_{1} \\
0 & -\lambda_{2}
\end{array}\right], & T_{1 o}=\left[\begin{array}{c}
(1-\alpha) \lambda_{1} \\
\lambda_{2}
\end{array}\right] \\
T_{2}=\left[\begin{array}{cc}
-\gamma_{1} & \beta \gamma_{1} \\
0 & -\gamma_{2}
\end{array}\right], & T_{2 o}=\left[\begin{array}{c}
(1-\beta) \gamma_{1} \\
\gamma_{2}
\end{array}\right]
\end{array}
$$

Notice that $T_{i}$ matrices correspond to phase transitions and sojourn times that do not result in arrivals. Similarly $T_{i o}$ entries correspond to the rate at which arrivals occur in the different phases. The service distributions are also assumed to be Coxian 2 with parameters: $\left(\mu_{1}, \delta, \mu_{2}\right)$ (note identical servers) $\left(\underline{e}_{1}^{T}, S_{1}, S_{1 o}\right)$ each with characteristics

$S_{1}=\left[\begin{array}{cc}-\mu_{1} & \delta \mu_{1} \\ 0 & -\mu_{2}\end{array}\right], \quad S_{1 o}=\left[\begin{array}{c}(1-\delta) \mu_{1} \\ \mu_{2}\end{array}\right]$

which are analogous to $T_{i}$ and $T_{i o}$ for the arrival process.

Now that we have described what is given in the problem, our next objective is to describe the main aim of this paper. Given the description of the queueing system scenario, our objective is to obtain the marginal distribution of the inter-departure time and its moments as well as the lag correlations between successive departures in the system. For this, we next model the system as a continuous time Markov chain (or Markov process) where the multidimensional states correspond to the number of jobs in the system, the phase of the first arrival stream, the phase of the second arrival stream and the phase of each server (if they are busy). The generator for the Markov process has the structure:

$Q=$\begin{tabular}{c|cccccc} 
& $\underline{0}$ & $\underline{1}$ & $\underline{2}$ & $\underline{3}$ & $\underline{4}$ & $\cdots$ \\
\hline$\underline{0}$ & $B_{00}$ & $C_{01}$ & $\underline{0}$ & $\underline{0}$ & $\underline{0}$ & \\
$\underline{1}$ & $A_{10}$ & $B_{11}$ & $C_{12}$ & $\underline{0}$ & $\underline{0}$ & \\
$\underline{2}$ & $\underline{0}$ & $A_{21}$ & $B$ & $C$ & $\underline{0}$ & $\cdots$ \\
$\underline{3}$ & $\underline{0}$ & $\underline{0}$ & $A$ & $B$ & $C$ & \\
$\vdots$ & & $\vdots$ & & $\ddots$ & $\ddots$ & $\ddots$
\end{tabular}

where the sub-matrices are defined in terms of Kronecker sum and product matrix operations as described in the next subsection. The states vary according to the number of customers in the system. For a system with two distinct arrival streams and two identical servers and assuming all processes are described by Coxian- 2 distributions, there are four states describing the arrival system and thus the $\underline{0}$ states have dimension $2^{2}$. The states for one customer in the system, denoted by 1 above, have dimension $2^{3}$ and the states for two, three, etc., customers in the system have dimensions $2^{4}$. The question is what are the values of $B_{00}$, $C_{01}, A_{10}, B_{11}, C_{12}, A_{21}, B, C$ and $A$ ? That is precisely what we will address next.

\subsection{Kronecker Matrix Operations}


Since the submatrices $B_{00}, C_{01}, A_{10}, B_{11}, C_{12}, A_{21}, B, C$ and $A$, described in the generator matrix $Q$ are obtained as Kronecker sums and products, it may be worthwhile to explain them briefly. The Kronecker product of any two matrices $L$ and $M$ results in matrix $K$ given by:

$$
K=L \otimes M=\left[l_{i j} M\right]
$$

Likewise the Kronecker sum of two matrices $L$ and $M$ yield matrix $K$ given as:

$$
K=L \oplus M=L \otimes I_{M}+I_{L} \otimes M
$$

where the identity matrices $I_{L}$ and $I_{M}$ correspond to the sizes of matrices $L$ and $M$, respectively.

\subsection{Model Matrix Elements}

Having defined the Kronecker product and sum, the next step is to use them to describe the matrices $B_{00}, C_{01}, A_{10}$, $B_{11}, C_{12}, A_{21}, B, C$ and $A$. For that we begin by defining $I_{T}$ as an identity matrix of dimensions equal to the sum of the dimensions of the two arrival processes (here there are two arrival streams each with dimension 2). Hence, $I_{T}=I_{4 \times 4}$. Likewise, $I_{S S}$ is an identity matrix of dimensions equal to the sum of the dimensions of the two service processes (here there are two identical servers each with dimension 2). Hence, $I_{S S}=I_{S} \oplus I_{S}=I_{4 \times 4}$.

Next we obtain submatrices $B_{00}, C_{01}, A_{10}, B_{11}, C_{12}, A_{21}$, $B, C$ and $A$, using the arrival and service process parameters in terms of the Kronecker product and sum. Specifically,

$$
A_{10}=I_{T} \otimes S_{1 o}
$$

and

$$
A_{21}=I_{T} \otimes\left(S_{1 o} \oplus S_{1 o}\right)
$$

The remaining submatrices can be obtained as follows. We use the notation $e_{i}^{T}$ to denote a unit (row) vector with a 1 in the $\mathrm{i}^{\text {th }}$ position. Let $S_{o}=S_{1 o} \otimes \underline{e}_{1}^{T}$, then

$$
\begin{aligned}
& A=I_{T} \otimes\left(S_{o} \oplus S_{o}\right) \\
& C_{01}=\left(\left(T_{1 o} \otimes \underline{e}_{1}^{T}\right) \oplus\left(T_{2 o} \otimes \underline{e}_{1}^{T}\right)\right) \otimes \underline{e}_{1}^{T} \\
& C_{12}=\left(\left(T_{1 o} \otimes \underline{e}_{1}^{T}\right) \oplus\left(T_{2 o} \otimes \underline{e}_{1}^{T}\right)\right) \otimes I_{S} \otimes \underline{e}_{1}^{T}
\end{aligned}
$$

$$
\begin{aligned}
& C=\left(\left(T_{1 o} \otimes \underline{e}_{1}^{T}\right) \oplus\left(T_{2 o} \otimes \underline{e}_{1}^{T}\right)\right) \otimes I_{S S} \\
& B_{00}=T_{1} \oplus T_{2} \\
& B_{11}=T_{1} \oplus T_{2} \oplus S_{1} \text { and } \\
& B=T_{1} \oplus T_{2} \oplus S_{1} \oplus S_{1}
\end{aligned}
$$

Once we have our $Q$ matrix the next step is to obtain some steady state results. The steady-state probabilities for this queue satisfy $\underline{\pi}^{T} Q=\underline{0}, \underline{\pi}^{T} \underline{1}=1$, and $\underline{\pi} \geq \underline{0}$, where we have partitioned $\underline{\pi}$ into blocks corresponding to the states for 0 jobs, 1 job, 2, jobs, etc., in the system. Hence, $\underline{\pi}^{T}=\left(\underline{p}_{0}^{T}, \underline{p}_{1}^{T}, \underline{p}_{2}^{T}, \cdots\right)$. Using the block probabilities and the elements of the $Q$ matrix, we need to find the $\underline{p}_{i}$ 's that satisfy:

$$
\begin{aligned}
& \underline{p}_{0}^{T} B_{00}+\underline{p}_{1}^{T} A_{10}=\underline{0} \\
& \underline{p}_{0}^{T} C_{01}+\underline{p}_{1}^{T} B_{11}+\underline{p}_{2}^{T} A_{21}=\underline{0} \\
& \underline{p}_{1}^{T} C_{12}+\underline{p}_{2}^{T} B+\underline{p}_{3}^{T} A=\underline{0} \\
& \underline{p}_{2+i}^{T} C+\underline{p}_{3+i}^{T} B+\underline{p}_{4+i}^{T} A=\underline{0} \quad(i=0,1,2, \cdots)
\end{aligned}
$$

Using (4), the matrix geometric procedure gives the vector solution $\underline{p}_{i}^{T}=\underline{p}_{2}^{T} R^{i-2}$, for $i=3,4,5, \cdots$, where $R$ is the matrix solution of the equation $C+R B+R^{2} A=\underline{0}$. Nuets (1981) showed that the iteration

$$
R_{k}=-\left(C+R_{k-1}^{2} A\right) B^{-1}
$$

converges to the solution $R$ starting with $R_{0}=\underline{\underline{0}}$. What remains are equations (1), (2) and (3) in $\operatorname{dim}\left(\underline{p}_{0}^{T}, \underline{p}_{1}^{T}, \underline{p}_{2}^{T}\right)$ unknowns and the norming equation $\underline{\pi}^{T} \underline{1}=1$. Solving these equations yields the steady state probability vector $\underline{\pi}$. Having obtain the steady state probabilities of various states of the queueing system, the next step is to use that describing the departure process from the queue. This is explained next.

\section{INTER-DEPARTURE TIMES}

When analyzing a network of queues, the output from some of the queues becomes inputs to others. So it is fre- 
quently necessary to characterize the departure process from a queue. The standard procedure for this analysis is to develop a renewal approximation for the output stream characterized by its mean and squared coefficient of variation (SCV) (Whitt 1983). Thus each departing job is assumed to have the same inter-departure time characteristics. The departure stream from queues with multiple input streams and/or multiple servers are intuitively not renewal processes. So we develop a generator for the steady-state departure process. From this analysis, the process moments as well as lag correlations can be obtained (see Bitran and Dasu 1994 for the single server queue analysis).

Characterizing the departure process involves developing a generator for the inter-departure times. The elements needed in this development are the departure-point statestate probabilities, $\underline{\delta}^{T}=\left(\underline{d}_{0}^{T}, \underline{d}_{1}^{T}, \underline{d}_{2}^{T}, \cdots\right)$. These are related to the continuous time steady-state probabilities $\underline{\pi}^{T}=\left(\underline{p}_{0}^{T}, \underline{p}_{1}^{T}, \underline{p}_{2}^{T}, \cdots\right)$ by the following relationships (here we denote the total arrival rate by $\bar{\lambda}$ due to superpositioning of the two Coxian arrival streams):

$$
\begin{aligned}
& \underline{d}_{0}^{T}=\underline{p}_{1}^{T} A_{10} / \bar{\lambda} \\
& \underline{d}_{1}^{T}=\underline{p}_{2}^{T} A_{21} / \bar{\lambda} \\
& \underline{d}_{n-1}^{T}=\underline{p}_{n}^{T} A / \bar{\lambda}, \quad n=3,4, \cdots
\end{aligned}
$$

The departure process consists of three distinct partitions: when there are no customers in the system, when there is one customer in the system and when there are two or more customers in the system. These three situations correspond to distinct characteristics for the departure process. When there are no customers in the system, a departure must wait until at least one arrival has occurred followed by a service. If a departing customer leaves the system with one customer in it, then the inter-departure time can be a function of the single processing customer's remaining service or it could evolve from an arrival and its completion of service. The third situation is when a departing customer leaves the system with at least 2 remaining customers and then the minimum of the remaining service time of one of these customers and the complete service time of another customer becomes the interdeparture time. Note that when there remain at least two customers in the system, the inter-departure time characteristics are the same for all these cases $(n \geq 2)$. The generator matrix for the departure process $\mathrm{G}$ has three distinct segmentations given by:

$$
G=\begin{array}{c|ccc} 
& \underline{0} & \underline{1} & \underline{2}^{+} \\
\hline \underline{0} & B_{00} & C_{01} & \underline{0} \\
\underline{1} & \underline{0} & B_{11} & \tilde{\tilde{C}}_{12} \\
\underline{2}^{+} & \underline{0} & \underline{0} & S S_{i n}
\end{array}
$$

where only two of these sub-matrices are new and are given by

$$
\begin{aligned}
& S S_{i n}=S_{1} \oplus S_{1} \\
& \tilde{C}_{12}=C_{12} \cdot\left(\underline{1} \otimes I_{T}\right)
\end{aligned}
$$

The probabilities of the departure-process system starting in the various states $\underline{\theta}=\left(\underline{d}_{0}^{T}, \underline{d}_{1}^{T}, \underline{d}_{2^{+}}^{T}\right)$ are made up of the departure point probabilities, with

$$
\underline{d}_{2^{+}}^{T}=\left(\sum_{n=3}^{\infty} \underline{p}_{n}^{T} A / \bar{\lambda}\right) \cdot\left(\underline{1} \otimes I_{T}\right)
$$

This series can be written in closed form as

$$
\begin{aligned}
\underline{d}_{2^{+}}^{T} & =\left(\sum_{n=3}^{\infty} \underline{p}_{2}^{T} R^{n-2} A / \bar{\lambda}\right) \cdot\left(\underline{1} \otimes I_{T}\right) \\
& =\left(\underline{p}_{2}^{T}\left[(I-R)^{-1}-I\right] A / \bar{\lambda}\right) \cdot\left(\underline{1} \otimes I_{T}\right)
\end{aligned}
$$

Using the above generator and truncated departure point probabilities, next we obtain the two main results of this paper, namely, the moments of the inter-departure times, and the lag correlations.

\subsection{Moments of inter-departure times}

Define $G^{\prime}$ as a single column matrix with each row element being equal to the negative of the sum of the corresponding row elements of $G$, then the stationary interdeparture time is a phase type distribution characterized by $\left[\underline{\theta}, G, G^{\prime}\right]$. Thus, from Neuts (1981) we know that the moments of inter-departure time random variable $X$ are given by

$$
E\left[X^{n}\right]=n !(-1)^{n} \underline{\theta}^{T}(G)^{-n} \underline{1}, \quad n=1,2, \cdots
$$

\subsection{Lag correlations between successive departures}

To obtain the lag- $k$ correlations of the output interdeparture times, it is necessary to develop the generator 
matrix of the background continuous time Markov chain $\tilde{G}$ segmented into two matrices: the internal transitions (without departures) $G$ (described above) and the matrix containing the departure transitions $G_{1}$ such that $\tilde{G}=G+G_{1}$. Notice that $G^{\prime}=G_{1} \cdot \underline{1}$ which is described in Section 3.1. The matrix $G_{1}$ is approximately characterized as

$$
G_{1}=\begin{array}{c|ccc} 
& \underline{0} & \underline{1} & \underline{2}^{+} \\
\hline \underline{0} & \underline{0} & \underline{0} & \underline{0} \\
\underline{1} & A_{10} & \underline{0} & \underline{0} \\
\underline{2}^{+} & \underline{0} & (1-p) \tilde{A}_{21} & p S S_{\text {out }}
\end{array}
$$

where $p$ is the probability that a departure leaves the system with at least two customers remaining and $(1-p)$ is the probability that only one customer remains. The matrix $\tilde{A}_{21}$ contains the expansion necessary to re-establish the arrival phase distribution associated with a single customer in service (that is, expanding the condensed states of $\underline{2}^{+}$ into the full state space of $\underline{1}$ ). The development of $\tilde{A}_{21}$ proceeds are follows. The steady-state probabilities of the states of the arrival process, $\underline{\theta}$, are obtained from its Markov arrival process representation

$$
\begin{aligned}
& \underline{\theta}^{T}\left(B_{00}+B_{i n}\right)=\underline{0}^{T} \\
& \underline{\theta}^{T} \underline{1}=1
\end{aligned}
$$

where the state changes associated with arrivals matrix $B_{\text {in }}$ is

$$
B_{i n}=\left(T_{1 o} \otimes \underline{e}_{1}^{T}\right) \oplus\left(T_{2 o} \otimes \underline{e}_{1}^{T}\right)
$$

and then

$$
\tilde{A}_{21}=\underline{\theta}^{T} \otimes\left(S_{1 o} \oplus S_{1 o}\right)
$$

Given the $G$ and $G_{1}$ matrices, from Telek and Horvath (2007) and Bodrog, Horvath and Telek (2008), we have that the lag- $k$ correlation is computed by

$$
\rho_{k}=\frac{\left(\sum \lambda_{i}\right)^{2} \underline{\theta}(-G)^{-1}\left((-G)^{-1} G_{1}\right)^{k}(-G)^{-1} \underline{1}-1}{2\left(\sum \lambda_{i}\right)^{2} \underline{\theta}(-G)^{-2} \underline{1}-1}
$$

Having obtained the moments of the marginal distributions of the inter-departure times from a queue as well as the lag- $k$ correlations, in the next section we provide a numerical example to illustrate the methodology and the results. However before that it may be worthwhile to make a connection between the output process and the parameters of the arrival process of a downstream node.

\subsection{Output as input to a downstream node}

We next briefly describe how this characterization of the output process in terms of the moments of inter-departure time as well as lag- $k$ correlations will be useful in modeling the input to a downstream node.

Since we use a phase-type or Coxian distribution for the inter-arrival time, it is crucial to determine the parameters of that distribution for a downstream node's arrival which directly corresponds to the output of the queue we have analyzed. Traditionally one matches moments which assumes that the departures are i.i.d. and ignores the correlations. Therefore to obtain a more robust set of parameters the idea is to match both the moments as well as the lag correlations. It is relatively straightforward to obtain the moments and lag correlations for a phase-type or Coxian arrival process. Then one has to select parameters so that the sum of squared deviation from the true value is minimized.

\section{EXAMPLE}

In this section we present a numerical example to illustrate the methodology and describe the results. Refer to section 2 for detailed notation. We begin by describing the two arrival processes $\left(\lambda_{1}, \alpha, \lambda_{2}\right),\left(\gamma_{1}, \beta, \gamma_{2}\right)$ along with the mean and SCV of the two streams as:

$$
\begin{aligned}
& \text { Arr 1:(20,0.25,5) } E\left[T_{a_{1}}\right]=0.1, \quad C_{a_{1}}^{2}=2 \\
& \operatorname{Arr} 2:(9.091,0.9,10) \quad E\left[T_{a_{2}}\right]=0.2, \quad C_{a_{2}}^{2}=0.55
\end{aligned}
$$

The composite mean time between arrivals $E\left[T_{a}\right]$ is $0.066 \overline{6}$ which is also equal to $1 / \bar{\lambda}$ defined earlier. And the phase representations of these two processes are:

$$
\begin{aligned}
& T_{1}=\left[\begin{array}{cc}
-20 & 5 \\
0 & -5
\end{array}\right], \quad T_{1 o}=\left[\begin{array}{c}
15 \\
5
\end{array}\right] \\
& T_{2}=\left[\begin{array}{cc}
-9.091 & 8.182 \\
0 & -10
\end{array}\right], \quad T_{2 o}=\left[\begin{array}{c}
0.909 \\
10
\end{array}\right]
\end{aligned}
$$


and the internal (non-arrival) phase changes for the composite arrival process are

$$
B_{00}=T_{1} \oplus T_{2}=\left[\begin{array}{cccc}
-29.091 & 8.182 & 5 & 0 \\
0 & -30 & 0 & 5 \\
0 & 0 & -14.091 & 8.182 \\
0 & 0 & 0 & -15
\end{array}\right]
$$

The phase changes associated with arrivals are:

$$
\begin{aligned}
B_{\text {in }} & =\left(T_{1 o} \otimes \underline{e}_{1}^{T}\right) \oplus\left(T_{2 o} \otimes \underline{e}_{1}^{T}\right) \\
& =\left[\begin{array}{cccc}
15.909 & 0 & 0 & 0 \\
10 & 15 & 0 & 0 \\
5 & 0 & 0.909 & 0 \\
0 & 5 & 10 & 0
\end{array}\right]
\end{aligned}
$$

The identical service processes $\left(\mu_{1}, \delta, \mu_{2}\right)$ are:

$$
(10,1 / 3,20), E\left[T_{S}\right]=0.11 \overline{6}, C_{S}^{2}=0.837
$$

along with the mean and SCV of the service times. In addition, similar to the arrival processes, we have

$$
S_{1}=\left[\begin{array}{cc}
-10 & 10 / 3 \\
0 & -20
\end{array}\right], \quad S_{1 o}=\left[\begin{array}{c}
20 / 3 \\
20
\end{array}\right]
$$

and thereby the internal phase changes are generated using the following matrix:

$$
S S_{\text {in }}=S_{1} \oplus S_{1}=\left[\begin{array}{cccc}
-20 & 10 / 3 & 10 / 3 & 0 \\
0 & -30 & 0 & 10 / 3 \\
0 & 0 & -30 & 10 / 3 \\
0 & 0 & 0 & -20
\end{array}\right]
$$

The associated departure phase matrix is

$$
S S_{\text {out }}=\left[\begin{array}{cccc}
40 / 3 & 0 & 0 & 0 \\
20 & 20 / 3 & 0 & 0 \\
20 & 0 & 20 / 3 & 0 \\
0 & 20 & 20 & 0
\end{array}\right]
$$

The corresponding service output matrix when the system transitions from two to one active servers is

$$
S_{1 o} \oplus S_{1 o}=\left[\begin{array}{cc}
40 / 3 & 0 \\
20 & 20 / 3 \\
20 & 20 / 3 \\
0 & 40
\end{array}\right]
$$

Now, using the definitions of $B_{00}, C_{01}, A_{10}, B_{11}, C_{12}$, $A_{21}, B, C$ and $A$ given in Section 2 for which all the input matrices are available numerically, we can obtain the infinitesimal generator matrix for the queue as

$$
Q=\begin{array}{c|cccccc} 
& \underline{0} & \underline{1} & \underline{2} & \underline{3} & \underline{4} & \cdots \\
\hline \underline{0} & B_{00} & C_{01} & \underline{0} & \underline{0} & \underline{0} & \\
\underline{1} & A_{10} & B_{11} & C_{12} & \underline{0} & \underline{0} & \\
\underline{2} & \underline{0} & A_{21} & B & C & \underline{0} & \cdots \\
\underline{3} & \underline{0} & \underline{0} & A & B & C & \\
\vdots & & \vdots & & \ddots & \ddots & \ddots
\end{array}
$$

The utilization factor for the system is 0.875 with a mean number of customers of 8.558 in the system in steady-state.

The steady-state probabilities $\underline{p}_{0}, \underline{p}_{1}, \underline{p}_{n}(n \geq 2)$ are of lengths 4,8 , and 16 , respectively. The $R$ and sub-matrices of $A, B, C$ of $Q$ are all of dimensions $16 \times 16$, so they won't be detailed here.

Next we describe numerical values and results for the terms defined in Section 3. The internal phase transition matrix component of the output generator process $G$ has no approximations involved and is straightforward to develop. To obtain the $G$ matrix, all the sub-matrices have either all been defined in $Q$ above or can be derived from the arrival and departure matrices using the expressions given in section 3. However, the departure phase matrix requires the expansion from the condensed two server active states denoted here-in by $\underline{2}^{+}$to the full arrival and serve states denoted by 1 . We approximate this expansion via the submatrix $\tilde{A}_{21}$. Let $\underline{\theta}$ be the solution to

$$
\begin{aligned}
& \underline{\theta}^{T}\left(B_{00}+B_{i n}\right)=\underline{0} \\
& \underline{\theta}^{T} \underline{1}=1,
\end{aligned}
$$

with $\underline{\theta}^{T}=(0.275,0.225,0.275,0.225)$. Then $\tilde{A}_{21}$ is obtained from $\underline{\theta}^{T} \otimes\left(S_{1 o} \oplus S_{1 o}\right)$ yielding 


$\left[\begin{array}{cccccccc}11 / 3 & 0 & 3 & 0 & 11 / 3 & 0 & 3 & 0 \\ 11 / 2 & 1.83 \overline{3} & 9 / 2 & 3 / 2 & 11 / 2 & 1.83 \overline{3} & 9 / 2 & 3 / 2 \\ 11 / 2 & 1.83 \overline{3} & 9 / 2 & 3 / 2 & 11 / 2 & 1.83 \overline{3} & 9 / 2 & 3 / 2 \\ 0 & 11 & 0 & 9 & 0 & 11 & 0 & 9\end{array}\right]$

The probability of a departure leaving the system with one job in service is obtained from the departure point probabilities. As these vectors has large dimensions and we need their sums rather than the individual vectors, we list these sum values only

$$
\underline{d}_{0}^{T} \underline{1}=0.0606, \underline{d}_{1}^{T} \underline{1}=0.0978, \sum_{n=1}^{\infty} \underline{d}_{n}^{T} \underline{1}=0.9394
$$

hence, $p=\underline{d}_{1}^{T} \underline{1} /\left(1-\underline{d}_{0}^{T} \underline{1}\right)=0.10407$. The resulting departure process characteristics are

$$
E\left[T_{d}\right]=0.066 \overline{6} \text {, and } C_{d}^{2}=0.9628
$$

and the lag- $k$ correlations 1-4 are:

$$
(0.00643,0.00497,0.00174,0.00023)
$$

Another method of estimating the probability breakout we can utilize the departure probability information and compute the ratio $\underline{\theta}$ from the departure vector $\underline{d}_{1}$. This approach yields

$$
\underline{\theta}^{T}=(0.2647,0.2335,0.2956,0.2061)
$$

which in turn yields a slightly different estimate of the lag$k$ correlations $1-4$ of

$$
(0.00660,0.00592,0.00279,0.00133)
$$

These two results are clearly close to one another, but neither one is exact. More work is needed to improve this approximation.

\section{CONCLUDING REMARKS AND FUTURE WORK}

In summary, in this paper we have considered a single node of a large queueing network. The main objective of our research is to characterize the departures from this multi-server queue (where service times according to phase-type distribution) with multiple phase-type streams superposed as arrival process. To illustrate our methodolo- gy we consider two independent Coxian-2 arrival streams that are fed into an infinite size queue with two identical servers. The service times for the servers are also according to Coxian-2 distribution. For this first come first served queueing system we describe the output process from the queue. Notice that the successive inter-departure times are correlated. Although most commonly the output is characterized by just the mean and SCV of the departure process, this study has shown that it is also possible to describe other output characteristics for fairly general settings such as the phase-type distribution. In particular, we obtain departure process characteristics such as by its distribution and moments as well as value of lag correlations. In a forthcoming journal paper we will describe the more generic case of phase type arrivals and service for the $N$ server queue. In addition, since the output from a queue would result in an input to a downstream node, we will provide an algorithm to use the moments and the lag correlations of the output process to fit the arrival process of a downstream node.

\section{REFERENCES}

Bitran, G. R., and S. Dasu. 1994. Analysis of the $\sum P h_{i} / P h / 1$ queue. Operations Research 42(1):158174.

Nuets, M. F. 1981. Matrix geometric solutions in stochastic models: an algorithmic approach. Baltimore: The John Hopkins. University Press.

Telek, M., and G. Horvath. 2007. A minimal representation of Markov arrival processes and a moments matching method. Performance Evaluation 64:1153-1168.

Bodrog L., A. Horvath, and M. Telek. 2008. Moment characterization of matrix exponential and Markovian arrival processes. Annals of Operations Research, to appear.

Whitt, W. 1983. The queueing network analyzer. The Bell System Technical Journal 62(9):2779-2814.

\section{AUTHOR BIOGRAPHIES}

GUY L. CURRY is a Professor of Industrial and Systems Engineering at Texas A\&M University. Dr. Curry received his B.S. in Mathematics from the University of Oklahoma, an M.S. degrees in Mathematics from Wichita State University, and the Ph.D. degree in Industrial Engineering from the University of Arkansas. His academic interests are optimization, simulation and queueing network approximations. Dr. Curry has published articles in over 30 referred journals and is the coauthor of three books.

NATARAJAN GAUTAM is an Associate Professor of Industrial and Systems Engineering at Texas A\&M University with a courtesy appointment in the department of 
Electrical and Computer Engineering. Dr. Gautam obtained his B.Tech. in Mechanical Engineering from the Indian Institute of Technology, Madras, and M.S. and Ph.D. degrees in Operations Research from the University of North Carolina at Chapel Hill. His research and teaching interests are design, control and performance evaluation of stochastic systems. Dr. Gautam is actively involved in service activities with INFORMS and IIE. 